\title{
Alveolar mechanics studied by in vivo microscopy imaging through intact pleural space
}

\author{
Caterina Salito ${ }^{\mathrm{a}, *}$, Andrea Aliverti $^{\mathrm{a}}$, Ilaria Rivolta ${ }^{\mathrm{b}}$, Enrico Mazzuca ${ }^{\text {, }}$ \\ Giuseppe Miserocchi ${ }^{\mathrm{b}}$
}

a Dipartimento di Elettronica, Informazione e Bioingegneria, Politecnico di Milano, Milano, Italy

${ }^{\mathrm{b}}$ Department of Experimental Medicine, University of Milano-Bicocca, Monza, Italy

\section{Introduction}

First attempts to investigate the mechanics of alveoli and alveolar ducts were based on quantitative analysis of histological samples fixated at various levels of inflation, but results were contrasting and strongly dependent on the fixation technique (Gatto et al., 2004). In vivo microscopy obviously represented a major advance to study alveolar mechanics (Carney et al., 1999; Liu et al., 2011; Tabuchi et al., 2008). So far, however, no relevance was given to potential interalveolar differences in mechanical properties; the main reason being the lack of resolution necessary to investigate this issue. This point actually received attention and represented the basis of a theoretical approach (Wilson, 2013) to estimate differences in elastic and surface components of alveolar distending pressure to justify differences in flow resistive properties. Aiming to pursue on this line of research, our group recently published a study based on in vivo imaging of unrestrained movement of sub pleural terminal alveolar units in static conditions (Mazzuca et al., 2014). The hypothesis arising from this work was that absolute alveolar compliance, $C_{\mathrm{abs}}$, increases with increasing alveolar size. The limitation of the study was that the same alveoli could not be followed on increasing alveolar pressure $\left(P_{\text {alv }}\right)$ and in fact information concerning change in size of alveoli was derived from the analysis of the cumulative frequency distribution of alveolar size at different distending pressure (Mazzuca et al., 2014). In order to test the validity of the above hypothesis, the aim of the present study was that to develop an experimental model to allow tracking, through in vivo imaging, of the same alveoli on increasing $P_{\text {alv }}$.

\section{Methods}

\subsection{Animal preparation}

A general consensus for the experimental procedures used in our research activity was obtained from the Local Ethical Committee. Experiments were performed on 6 adult (New Zealand White) male rabbits (weight range $1-1.5 \mathrm{~kg}$ ) anesthetized with a bolus of $2.5 \mathrm{ml} / \mathrm{kg}$ of a saline solution containing $0.25 \mathrm{~g} / \mathrm{ml}$ of urethane injected into an ear vein; anesthesia was maintained by repetitive injections of anesthetic $(0.5 \mathrm{ml})$ every $20 \mathrm{~min}$. Tracheostomy was performed, and a plastic cannula (length $50 \mathrm{~mm}$, inner diameter $2.5 \mathrm{~mm}$ ) was inserted into the distal trachea. Before connecting the animal to the ventilator, paralysis was accomplished by

\footnotetext{
* Corresponding author at: Dipartimento di Elettronica, Informazione e Bioingegneria, Politecnico di Milano, P.zza L. da Vinci, 32, 20133 Milano, Italy. Tel.: +3902 2399 9026; fax: +39022399 9000.

E-mail address: caterina.salito@polimi.it (C. Salito).
} 


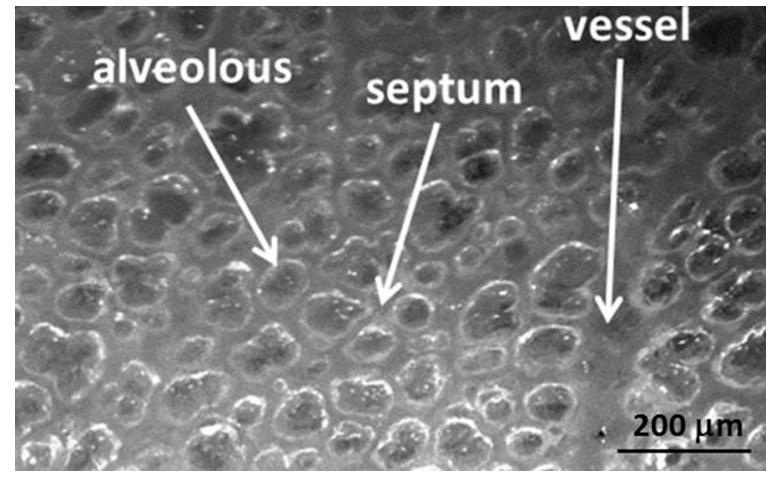

Fig. 1. Imaging of subpleural alveoli through a pleural window allowing a net view of alveoli, intervening septa and a micro vessel.

pancuronium bromide ( $1 \mathrm{mg} / \mathrm{kg}$ body weight initial dose, supplemented by $0.33 \mathrm{mg} / \mathrm{kg}$ every $40 \mathrm{~min}$ ). Mechanical ventilation was provided with a tidal volume of $\sim 20 \mathrm{ml}$ and a frequency of 16 breaths/min. An "intact pleural window" was prepared in the sixth intercostal space to allow a view of the lower lobe where the cardiac artifacts are relatively small. The skin and superficial muscles on the right side of the chest were resected and when reaching the layer of the intercostal muscles, a surface area of about $0.5 \mathrm{~cm}^{2}$ was freed from muscles down to the endothoracic fascia. Under stereomicroscopic view and with fine forceps, we then carefully stripped the endothoracic fascia, exposing the parietal pleura (about $10 \mu \mathrm{m}$ thick) over an average surface of about $2.5 \mathrm{~mm}^{2}$, through which the lung surface with its alveolar texture could be clearly detected. This approach allowed to neatly visualize the sub pleural alveoli freely moving with intact pleural space mechanics.

\subsection{Experimental protocol}

The animal was placed under the field of a fixed microscope (Nikon SMZ-2T) in left lateral decubitus; therefore, we imaged the change in size of alveoli of the caudal part of the right lower lobe where no interlobar fissures are present. Given the animal position in left lateral decubitus, the alveoli studied were in the less dependent portion of the pleural cavity. Images of the lung surface through the intact parietal pleura were acquired with a video camera (CoolSNAPEZ, Photometrics), connected to the microscope, interfaced through a IEEE-1394 data-transfer interface card with a personal computer equipped with an image-processing software (MetaMorph ${ }^{\circledR}$ System, Molecular Devices). Total microscopic magnification was $60 \times$. A LED ring-light illuminator was anchored to microscopic optics to provide a uniform lighting of the alveolar field. The displacement of the alveolar field under microscopic view required a manual focus adjustment at each pressure step.

\subsection{Image analysis}

The transparency of the parietal pleura allowed a clear identification of the alveolar texture; Fig. 1 shows an example of the morphology of the terminal alveolar units through the pleural window. Alveolar units, henceforth simply referred to as alveoli, both at 4 and $8 \mathrm{cmH}_{2} \mathrm{O}$, were manually delimited by following their borders well identified by the gray level gradient between air and tissue phase. Restricting the pressure range from 4 to $8 \mathrm{cmH}_{2} \mathrm{O}$ allowed us to follow the morphology of the same alveoli, that could be identified within the image field. Alveolar segmentation allowed to derive the alveolar 2D surface area. Alveolar aspect ratio (AR), as a shape factor, was defined as major diameter/minor diameter.

After stopping mechanical ventilation, lung volume was increased by increasing $P_{\text {alv }}$ from $4 \mathrm{cmH}_{2} \mathrm{O}$ up to $8 \mathrm{cmH}_{2} \mathrm{O}$, which allowed to estimate the alveolar areas corresponding to these two pressures, indicated as $A_{4}$ and $A_{8}$, by tracking the same alveoli.

We obtained $P_{\text {alv }}$ vs alveolar area relationships for alveoli identified within the imaging field. An index of absolute alveolar compliance was defined as $C_{\mathrm{abs}}=\left(A_{8}-A_{4}\right) /\left(P_{8}-P_{4}\right)$ furthermore we also expressed specific alveolar compliance as $C_{\mathrm{sp}}=C_{\mathrm{abs}} / A_{4}$.

At the end of the experiment, the animals were euthanized with an anesthetic overdose.

\subsection{Statistical analysis}

Quartile coefficient of variation (QVC), equal to the ratio between interquartile range and the sum of the 75th and the 25th percentiles, was calculated for $A_{4}, A_{8}, C_{\mathrm{abs}}$ and $C_{\mathrm{sp}}$ distributions. The effects of alveolar areas at different $P_{\mathrm{alv}}$ on $C_{\mathrm{abs}}$ and $C_{\mathrm{sp}}$ were tested by using a paired $t$-test. Univariate linear regression analysis was used to examine the relationship between $C_{\mathrm{abs}}$ and $A_{4}$. Oneway ANOVA analysis was performed to evaluate the effect of the alveolar size at $4 \mathrm{cmH}_{2} \mathrm{O}$ on $C_{\mathrm{sp}}$. A $t$-test was performed to evaluate the significance of differences in mean or median values. Statistical analysis was performed by SigmaStat software v11.0 (San Jose, CA, USA). All data are reported as mean values \pm standard error. $P$-values lower than 0.05 were considered to indicate significant differences.

\section{Results}

Fig. 2A reports the distribution of $A_{4}$ showing that the most abundant alveoli are those whose area was in the range $1000-3000 \mu \mathrm{m}^{2}$. Fig. 2B presents how the distribution was changed at pressure of $8 \mathrm{cmH}_{2} \mathrm{O}$. Both distributions were not normal with a QCV equal to 0.41 and 0.31 respectively. Fig. $3 \mathrm{~A}$ shows the result of the manual segmentation of single alveoli of different caliber at alveolar pressure of $4 \mathrm{cmH}_{2} \mathrm{O}$ and at $8 \mathrm{cmH}_{2} \mathrm{O}$. Fig. 3B shows the relationship of alveolar area vs $P_{\text {alv }}$ for the same alveoli shown in Fig. 3A; hysteresis for the distending pressures considered was minimal. The average slope of these relationships may be considered an index of the $C_{\mathrm{abs}}$, and the figure allows to appreciate that $C_{\mathrm{abs}}$ was greater the greater the value $A_{4}$. Fig. $4 \mathrm{~A}$ shows that $C_{\text {abs }}$ positively correlates significantly $(P=0.0001)$ with the value of $A_{4}$, with a $r^{2}=0.59$. Fig. $4 \mathrm{~B}$ shows the distribution of $C_{\mathrm{abs}}$ for all alveoli considered in the pressure range 4-8 $\mathrm{cmH}_{2} \mathrm{O}$; this distribution was not normal, with a skewness coefficient of 0.72 and a QVC of 0.49. Fig. 5A shows that the derived specific compliance $C_{\mathrm{sp}}$ did not show any specific trend of dependence on $A_{4}$, being therefore relatively independent on alveolar size. A considerable variability was present, being highest for alveolar area values in the range of $1000-3000 \mu \mathrm{m}^{2}$, that includes about $70 \%$ of alveoli. Fig. 5B shows the distribution of $C_{\mathrm{sp}}$ in box plots for three groups of $A_{4}$; by one-way ANOVA analysis, median values of $C_{\mathrm{sp}}$ were not statistically different among groups $(P=0.162)$ so that the overall $C_{\mathrm{sp}}$ median value of $0.21 \mathrm{cmH}_{2} \mathrm{O}^{-1}$ is shown in Fig. $5 \mathrm{~A}$ with 25 th and 75th percentiles (QVC equal to 0.22 )

Fig. 6 shows the AR for three groups of alveoli of different size at 4 (Fig. 6A) and 8 (Fig. 6B) $\mathrm{cmH}_{2} \mathrm{O}$. For alveoli of $0-3000 \mu \mathrm{m}^{2}$ the median values of AR (not normal distribution) increased significantly (from 1.13 to $1.28, P<0.001$ ) from 4 to $8 \mathrm{cmH}_{2} \mathrm{O}$. For alveoli $>5000 \mu \mathrm{m}^{2}$ the mean values of AR significantly decreased (from 1.67 to $1.40, P=0.018$ ). No significant changes in AR were observed for alveoli of $3000-5000 \mu \mathrm{m}^{2}$.

\section{Discussion}

In a previous study (Mazzuca et al., 2014), taking advantage of a technique allowing transpleural imaging of unrestrained alveolar 

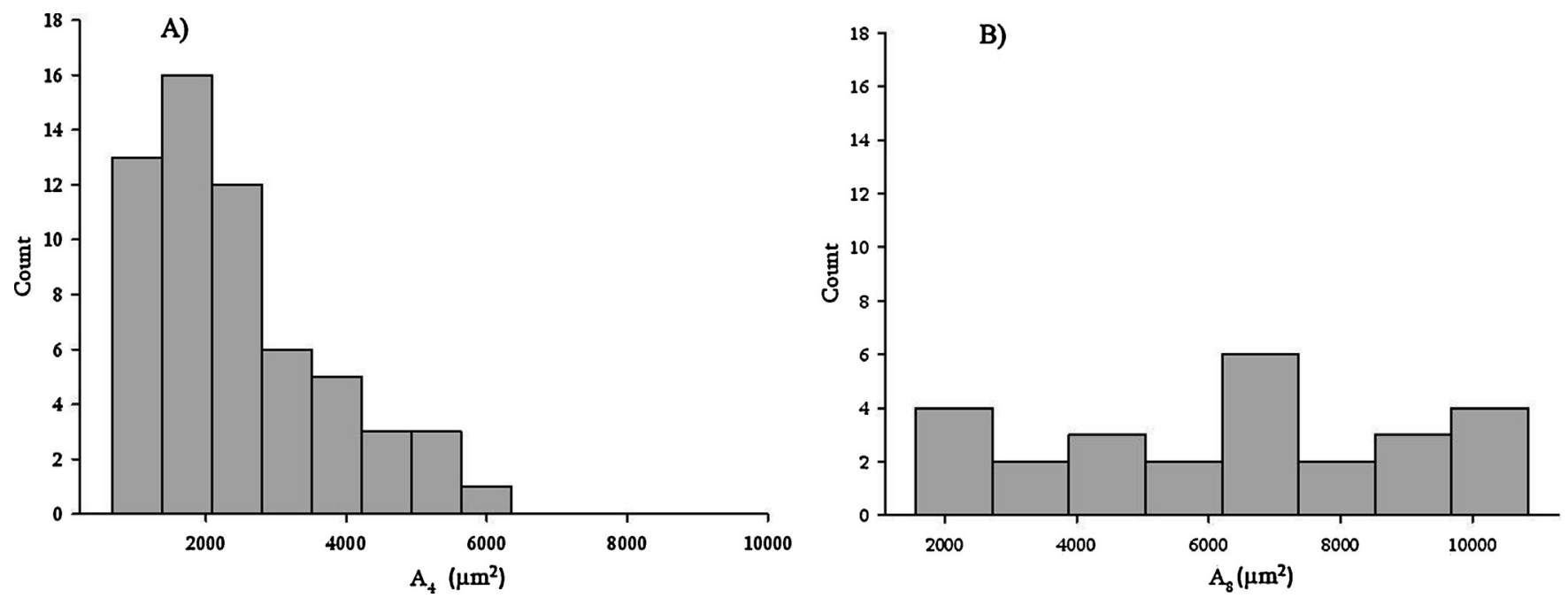

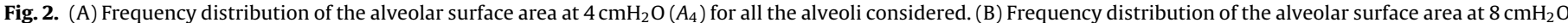
$\left(A_{8}\right)$ for all the alveoli considered.
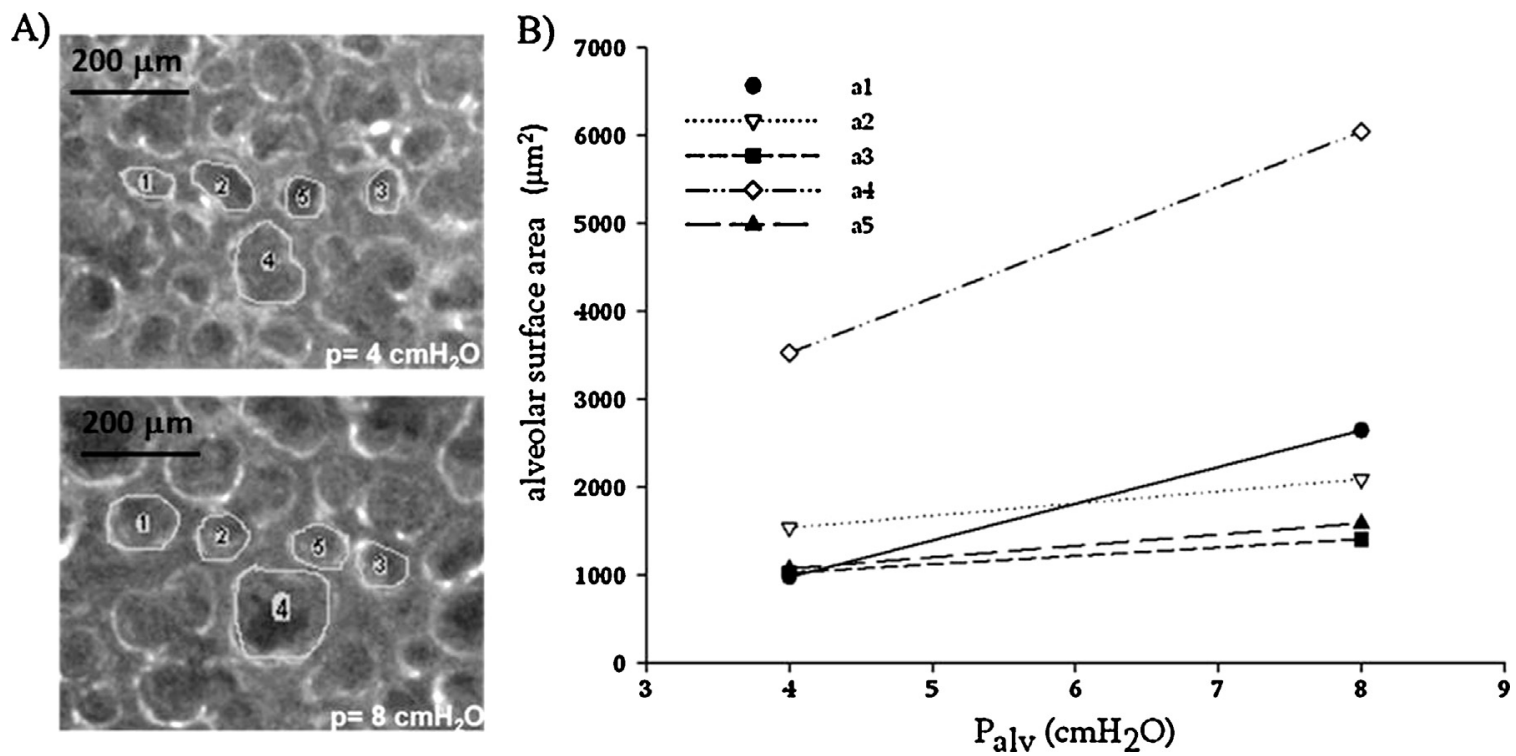

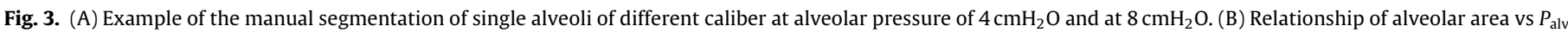
for the same alveoli shown in (A).
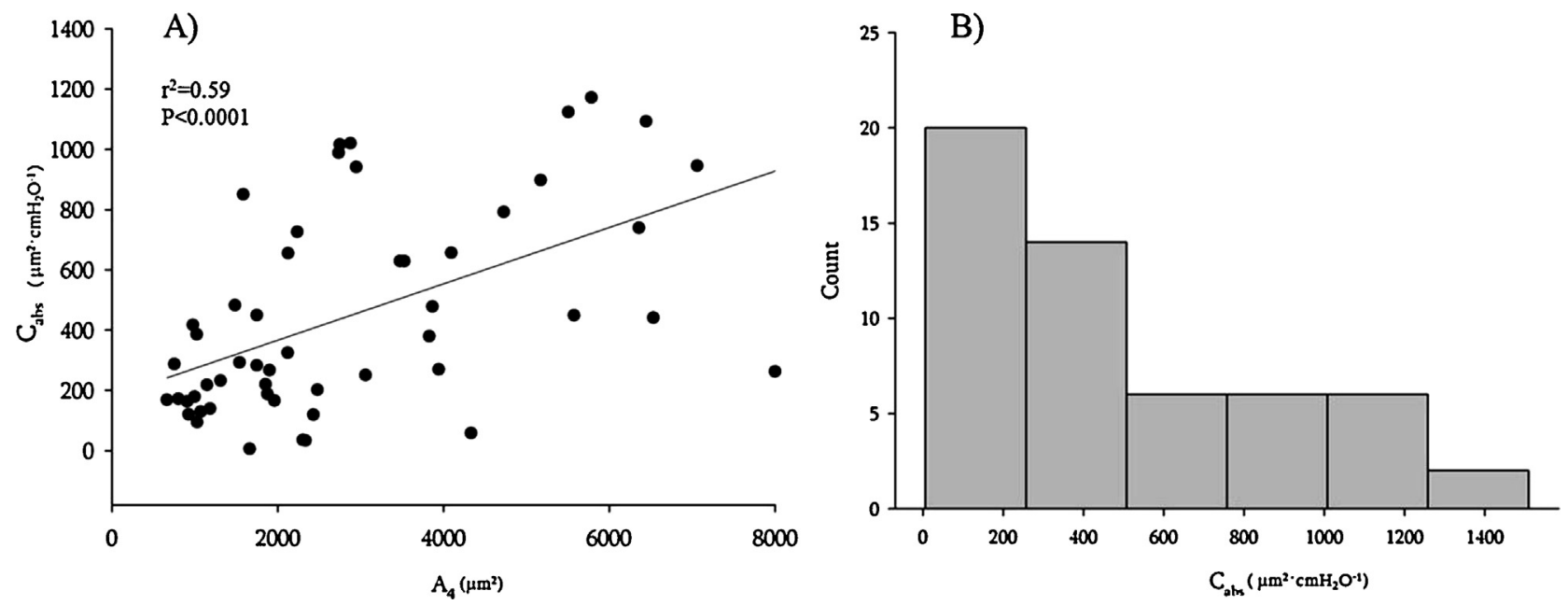

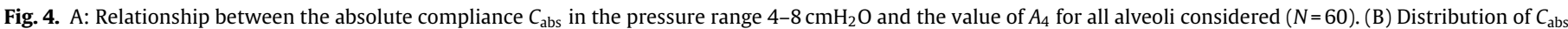
for all alveoli considered in the pressure range $4-8 \mathrm{cmH}_{2} \mathrm{O}$. 

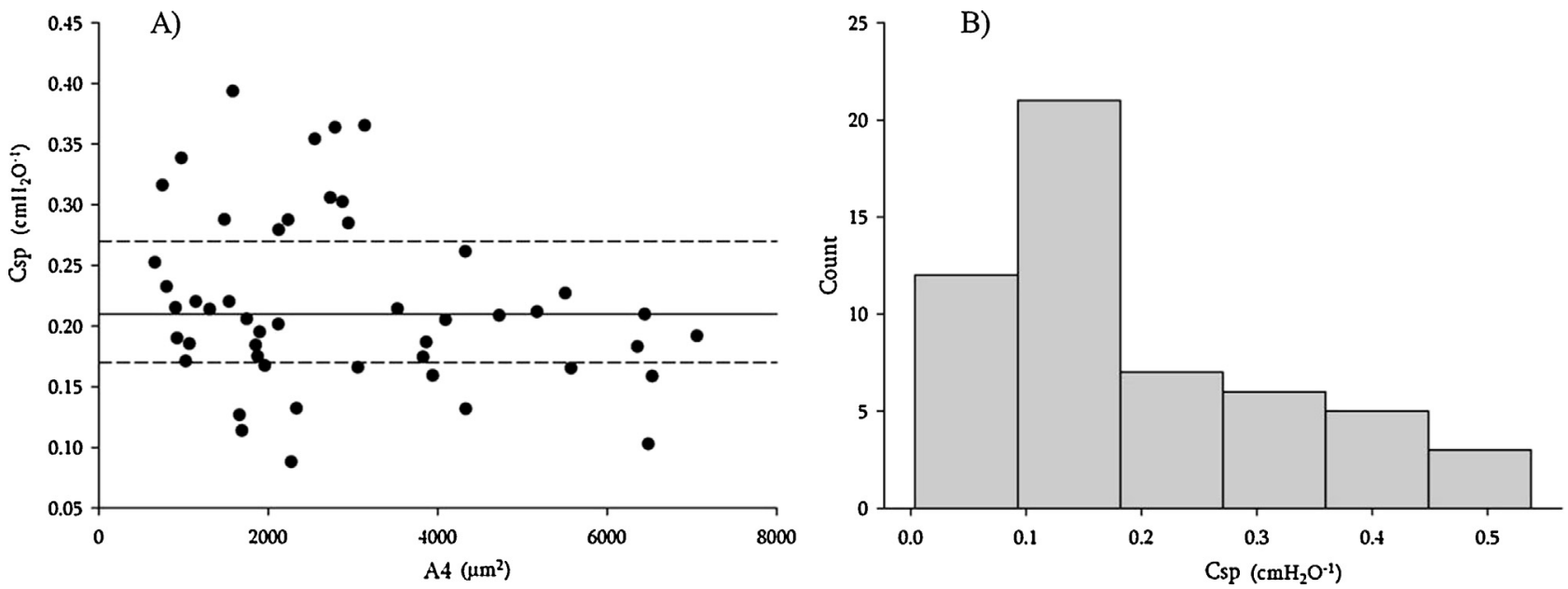

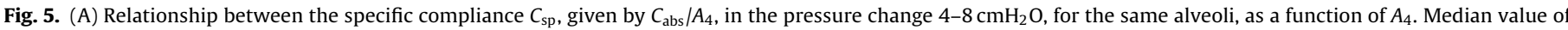

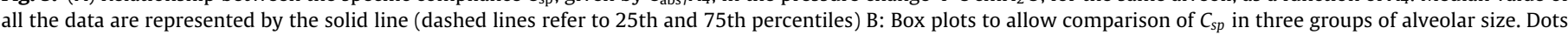
refer to 25 th and 75 th percentiles.

motion, considerable differences in the distribution of alveolar size on varying alveolar pressure were documented.

The present data confirm the heterogeneity in the distribution in alveolar size taking as a pre-stress conditions $P_{a l v}=4 \mathrm{cmH}_{2} \mathrm{O}$ (Fig. 2A). In addition, by following the same alveoli during inflation maneuver up to $8 \mathrm{cmH}_{2} \mathrm{O}$ (about 40\% increase in lung volume relative to $4 \mathrm{cmH}_{2} \mathrm{O}$ ), we now demonstrate that indeed $C_{\text {abs }}$ was greater the greater the alveolar radius (Fig. 4A) and its frequency distribution was not normal in physiological condition (Fig. 4B).

We show that $C_{\mathrm{abs}}$ increases by about one order of magnitude for alveoli from 1000 to $7000 \mu \mathrm{m}^{2}$. Yet, only $60 \%$ of the variance of $C_{\mathrm{abs}}$ could be explained by its dependence on alveolar size. Thus, other factors impact on $C_{\mathrm{abs}}$ such as, on one side, possible noise/errors due to image spatial resolution and manual segmentation (see below), on the other, morpho-functional differences related to alveolar geometry, hysteresis of the surface film, tissue-vascular/bronchi interactions and, finally, macro-molecular organization of the extra-cellular component.

The concept of having heterogeneous distribution of the alveolar mechanical properties has been put forward in experimental models causing a remarkable perturbation of the physiological state of the lung (Wilson, 2013), including inhomogeneous broncho-constriction (Davey and Bates, 1993; Suki et al., 1997), acutely injured lung (Schiller et al., 2001), injurious ventilation (Allen et al., 2005), elastase treatment to damage the elastic component (Ito et al., 2004). The mechanical changes were evaluated in terms of respiratory impedance, flow resistance, inertance, viscous energy dissipation and energy storage (Bates and Suki, 2008).

The present data were gathered by preserving the integrity of the pleural space and therefore the physiological condition of lung distension and free movement. Since we referred to quasi static conditions, flow resistance and inertance were nil and therefore the inter-alveolar differences in $C_{\mathrm{abs}}$ may be interpreted in terms of elastic (including energy dissipation and energy storage) and surface alveolar forces. However, although our condition may be considered static for elastic forces, this may not necessarily be the case for alveolar surface tension because of the driving force for surfactant redistribution due to Marangoni effect.

\subsection{Limitations of the study}

The advantage offered by our protocol was that to study alveolar dynamics by imaging of the same alveoli, with freely moving lung by preserving the integrity of the pleural space. Such advantage

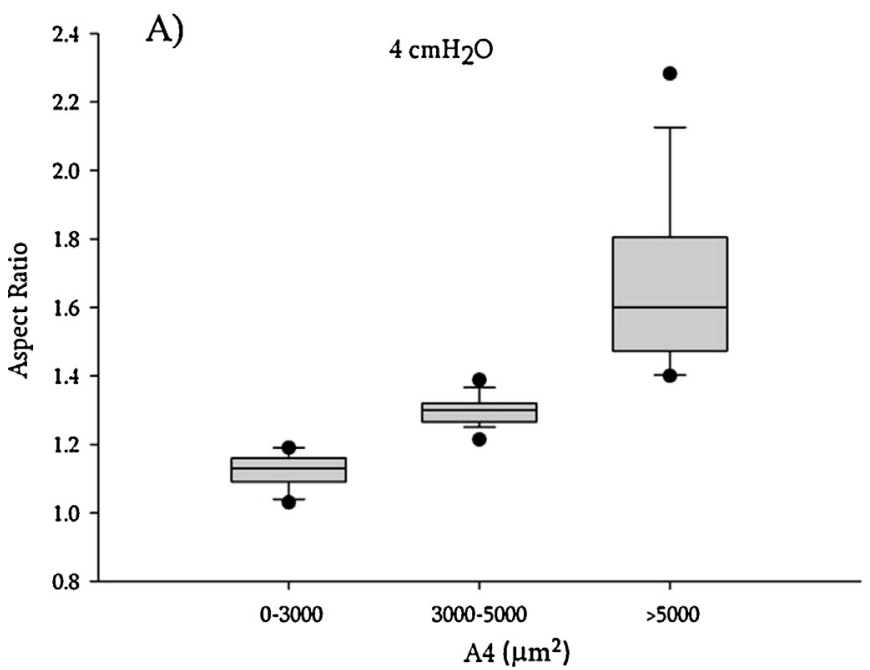

B)

\section{$8 \mathrm{cmH}_{2} \mathrm{O}$}
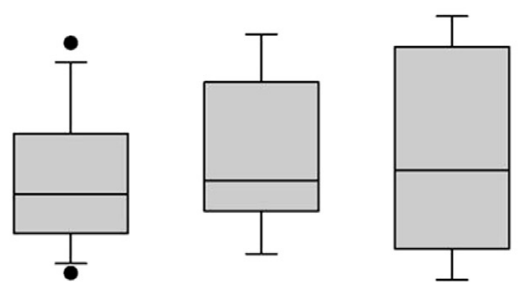

$0-3000$

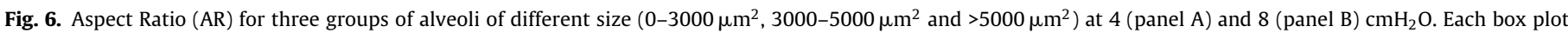
shows median, 25th-75th and 5th-95th percentiles. 
was not obvious in other preparation (Mertens et al., 2009). On the other hand, our technique suffers from sampling limitations because it is restricted to the study of the group of alveoli within the pleural window that can be tracked on changing distending pressure. The images on which we performed the analysis refer to acinar structures belonging to terminal units, however, an obvious difficulty in performing the segmentation of terminal airways units is due to their morphology that varied from single alveoli to seemingly assembly of alveoli. This study reveals differences in alveolar mechanics related to heterogeneity in alveolar size based on 2D microscopic images that should actually, in part reflect also the heterogeneity in the 3D shapes. Assuming isotropic lung expansion, the changes in areas of the acinar structures are numerically related to the changes in volume. Another potential limitation is represented by the fact that only subpleural units were studied and therefore we are not entitled to extend the observations to the rest of the lung, although no differences in surface area expansion were reported for subpleural and core alveoli from fixed rabbit lungs (Gil et al., 1979).

\subsection{From absolute to specific alveolar compliance}

Despite the limitations of the study, the present data may offer the clue to discuss about the fact that a difference in mechanical properties of alveoli, as reflected by the differences in $C_{\mathrm{abs}}$, would cause distortion of the surrounding alveoli due to interdependence. In general, differences in the contribution of surface tension and of local elastic distending pressure $\left(P_{\gamma}\right.$ and $P_{\mathrm{el}}$, respectively) to total distending pressure $\left(P_{\mathrm{ld}}\right)$, for $P_{\mathrm{ld}}=P_{\gamma}+P_{\mathrm{el}}$, have been considered. Differences in $P_{\gamma}$ relating to differences in alveolar expansion may be envisaged due to the dependence of surface tension $\gamma$ on alveolar surface (Gil et al., 1979). The hysteresis of the surface film during small-amplitude volume changes, as in our case, was con-

sidered negligible (Schurch, 1982). Differences in $P_{\mathrm{el}}$ may result from the differences in force vs extension ratio of parenchymal tissue surrounding the alveoli (Wilson and Bachofen, 1982) due to different contents in the fibrillar component that includes collagen and elastin and is mostly responsible for elastic behavior. Concerning the non-fibrillar component, including the waste proteoglycans family, one may hypothesize local differences in viscoelastic properties that critically depend upon the extravascular water content (Cavalcante et al., 2005; Miserocchi et al., 2001). The concept of specific compliance has been developed for the whole lung as a ratio of the absolute compliance to lung volume: it was found that, although absolute lung compliance did increase with increase in body size, specific lung compliance was essentially independent on body size (Crosfill and Widdicombe, 1961). This feature was interpreted considering that absolute compliance reflected the total mass of lung tissue and therefore the number of alveolar units that obviously increases with body size. We now propose to interpret

the differences in alveolar $C_{\mathrm{abs}}$ as reflecting the tissue mass of which an alveolar wall is made. Indeed, on the assumption that the alve-olar wall contains a network of interconnected elements of equal elastic properties, it appears reasonable to derive that alveolar com-pliance is increasing with increasing number of the elastic elements as it is the case with increasing alveolar size. The present data confirm the hypothesis derived in a previous study (Mazzuca et al., 2014) for the relatively constancy of $C_{\mathrm{sp}}$ up to a distending pres-sure of $16.5 \mathrm{cmH}_{2} \mathrm{O}$, thus over a much wider range of distending pressure relative to the present study.

This interpretation is in accordance with the mechanical model proposed by Kimmel et al. (Kimmel et al., 1987). These authors normalized the strain to the initial length of the force bearing elements as well as the surface tension to the values occurring in the pre-stress conditions.

\subsection{Alveolar mechanical stability}

One may hypothesize that on increasing alveolar distension, dif-ferences in acting forces might generate between small and large alveoli implying different contribution of $P_{\gamma}$ and $P_{\mathrm{el}}$ Indicatively, changes in $P_{\gamma}$ are likely more important than changes in $P_{\mathrm{el}}$ as the former may increase about fivefold in the alveolar volume range 40-60\% TLC (Wilson, 1982) while the latter essentially doubles.

This factor may be considered to explain the differences in AR as a function of alveolar size (Fig. 6) considering that a greater AR value is an index of greater deformability. We found that at low dis-tending pressure, the alveolar AR increased with increasing alveolar size, suggesting a greater deformability of larger alveoli that can be justified on the basis of greater absolute compliance (Fig. 4A). At the higher distending pressure AR was found to decrease for larger alveoli but to increase for the smaller ones, thus deformabil-ity decreased for larger alveoli but increased for smaller ones. To explain this finding one may hypothesize the following sequence:(a) the greater increase in volume for larger alveoli would cause a greater thinning of the surfactant film and, correspondingly, some increase in surface tension resulting in less deformability, (b) this effect would be buffered by surfactant redistribution (Marangoni flow) from smaller alveoli that, being deprived of surfactant, would then become more rigid. This sequence may be hypothesized considering that Marangoni flows are based on surface shear of the surfactant in a liquid expanded state, as it is supposed to be in the lung, although it is not certain the extent to which surfactant viscosity is limiting its flow (Kim et al., 2011; Hermans and Vermant, 2014). There are therefore indications that, on increasing lung vol-ume, there are differences in elastic and surface forces within the alveolar walls depending on alveolar size. We found that $C_{\mathrm{sp}}$ was essentially independent of alveolar size and consider this feature as limiting interregional differences in parenchymal and surface forces, thus contributing to assure a fairly uniform stretching in a model of mechanically interdependent alveoli. Yet, $C_{\mathrm{sp}}$ showed a fourfold scatter, which could be accounted for by the size-related

inter-alveolar differences in $P_{\gamma}$ and $P_{\mathrm{el}}$ on increasing lung volume, as discussed above.

In summary, we are dealing with a large alveolar geometric heterogeneity but a somehow less variable specific mechanical homogeneity. As suggested by a recent publication (Mazzuca et al., 2014), it is interesting to recall that the non-topological dependent morphological and mechanical heterogeneity at alveolar level results in a relatively homogeneous mechanical behavior of the whole lung.

\section{Acknowledgement}

This work was funded by Fondazione Cariplo.

\section{References}

Allen, G.B., Pavone, L.A., Di Rocco, J.D., Bates, J.H., Nieman, G.F., 2005. Pulmonary impedance and alveolar instability during injurious ventilation in rats. J. Appl. Physiol. 99 (August (2)), 723-730.

Bates, J.H., Suki, B., 2008. Assessment of peripheral lung mechanics. Respir. Physiol. Neurobiol. 163 (November (1-3)), 54-63.

Carney, D., Bredenberg, C., Schiller, H., Picone, A., McCann, U., Gatto, L., Bailey, G., Fillinger, M., Nieman, G., 1999. The mechanism of lung volume change during mechanical ventilation. Am. J. Respir. Crit. Care Med. 160, 1697-1702. Cavalcante, F.S., Ito, S., Brewer, K.K., Sakai, H., Alencar, A.M., Almeida, M.P., Andrade Jr., J.S., Majumdar, A., Ingenito, E.P., Suki, B., 2005. Mechanical interactions between collagen and proteoglycans: implications for the stability of lung tissue. J. Appl. Physiol. 98, 672-679.

Crosfill, M.L., Widdicombe, J.G., 1961. Physical characteristics of the chest and lungs and the work of breathing in different mammalian species. J. Physiol. 158 (September), 1-14.

Davey, B.L., Bates, J.H., 1993. Regional lung impedance from forced oscillations through alveolar capsules? Respir. Physiol. 91 (March (2-3)), 165-182. 
Gatto, L., Fluck, R., Nieman, G., 2004. Alveolar mechanics in the acutely injured lung: role of alveolar instability in the pathogenesis of ventilator-induced lung injury. Respir. Care 49, 1045-1055.

Gil, J., Bachofen, H., Gehr, P., Weibel, E.R., 1979. Alveolar volume-surface area relation in air- and saline-filled lungs fixed by vascular perfusion. J. Appl. Physiol. 47, 990-1001.

Hermans, E., Vermant, J., 2014. Interfacial shear rheology of DPPC under physiologically relevant conditions. Soft Matter 10 (1), 175-186.

Ito, S., Ingenito, E.P., Arold, S.P., Parameswaran, H., Tgavalekos, N.T., Lutchen, K.R. Suki, B., 2004. Tissue heterogeneity in the mouse lung: effects of elastase treatment. J. Appl. Physiol. 97 (July (1)), 204-212.

Kim, K., Choi, S.Q., Zasadzinski, J.A., Squires, T.M., 2011. Interfacial microrheology of DPPC monolayers at the air-water interface. Soft Matter 7 (17), 7782-7789.

Kimmel, E., Kamm, R.D., Shapiro, A.H., 1987. A cellular model of lung elasticity. J. Biomech. Eng. 109 (May (2)), 126-131.

Liu, H., Runck, H., Schneider, M., Tong, X., Stahl, C., 2011. Morphometry of subpleural alveoli may be biased by local pressure changes induced by the microscopic device. Respir. Physiol. Neurobiol. 178, 283-289.

Mazzuca, E., Salito, C., Rivolta, I., Aliverti, A., Miserocchi, G., 2014. From morphological heterogeneity at alveolar level to the overall mechanical lung behavior: an in vivo microscopic imaging study. Physiol. Rep. 2 (1).

Mertens, M., Tabuchi, A., Meissner, S., Krueger, A., Schirrmann, K., Kertzscher, U., Pries, A.R., Slutsky, A.S., Koch, E., Kuebler, W.M., 2009. Alveolar dynamics in acute lung injury: heterogeneous distension rather than cyclic opening and collapse. Crit. Care Med. 37 (September (9)), 2604-2611.

Miserocchi, G., Negrini, D., Passi, A., De Luca, G., 2001. Development of lung edema: interstitial fluid dynamics and molecular structure. News Physiol. Sci. 16 (April), 66-71 (Review)

Schiller, H.J., McCann 2nd, U.G., Carney, D.E., Gatto, L.A., Steinberg, J.M., Nieman, G.F., 2001. Altered alveolar mechanics in the acutely injured lung. Crit. Care Med. 29 (May (5)), 1049-1055.

Schurch, S., 1982. Surface tension at low lung volumes: dependence on time and alveolar size. Respir. Physiol. 48, 339-355.

Suki, B., Yuan, H., Zhang, Q., Lutchen, K.R., 1997. Partitioning of lung tissue response and inhomogeneous airway constriction at the airway opening. J. Appl. Physiol. 82 (April (4)), 1349-1359.

Tabuchi, A., Mertens, M., Kuppe, H., Pries, A.R., Kuebler, W.M., 2008. Intravita microscopy of the murine pulmonary microcirculation. J. Appl. Physiol. 104 338-346.

Wilson, T.A., 1982. Surface tension-surface area curves calculated from pressure-volume loops. J. Appl. Physiol. Respir. Environ. Exerc. Physiol. 53 (December (6)), 1512-1520.

Wilson, T.A., Bachofen, H., 1982. A model for mechanical structure of the alveolar duct. J. Appl. Physiol. 52, 1064-1070.

Wilson, T.A., 2013. Parenchymal mechanics, gas mixing, and the slope of phase III. J. Appl. Physiol. 115 (July (1)), 64-70. 